\title{
Effects of Expectancy and Abstinence on the Neural Response to Smoking Cues in Cigarette Smokers: An fMRI Study
}

\author{
Dharma McBride', Sean P Barrett ${ }^{2}$, Jared T Kelly', Andrew Aw' and Alain Dagher*,' \\ 'McConnell Brain Imaging Center, Montreal Neurological Institute, McGill University, Montreal, QC, Canada; ${ }^{2}$ Department of Psychology, \\ McGill University, Montreal, QC, Canada
}

\begin{abstract}
Cues associated with drug taking can trigger relapse, drug seeking, and craving in addicted individuals. Behavioral studies suggest that drug availability and withdrawal can affect the individual response to drug cues. Moreover, the importance of subjective craving in cue-induced relapse has been questioned and an alternative model put forward according to which drug cues trigger habitual drug-seeking behaviors independently of craving. We used functional magnetic resonance imaging to compare the brain response to smoking and control videotapes in 20 healthy smokers, while varying their expectancy to smoke and abstinence levels. The neural response to cigarette cues was strongly modulated by expectancy and, to a lesser extent, abstinence. In people expecting to smoke immediately after the scan, smoking cues activated brain areas implicated in arousal, attention, and cognitive control. However, when subjects knew they would not be allowed to smoke for $4 \mathrm{~h}$, there was almost no brain activation in response to smoking cues, despite equivalent reported levels of craving. In the dorsolateral prefrontal cortex, the neural response was a function of both craving and expectancy. Thalamo-cingulate connectivity, thought to be an index of arousal, was greater during expectancy than nonexpectancy. Our findings confirm the importance of expectancy in the neural response to drug cues, and lend support to the theory that these cues act on brain areas involved in arousal and attention.

Neuropsychopharmacology (2006) 3 I, 2728-2738. doi:I0.1038/sj.npp. I301075; published online 5 April 2006
\end{abstract}

Keywords: drug cues; dorsolateral prefrontal cortex; anterior cingulate cortex; craving; functional magnetic resonance imaging; nicotine

\section{INTRODUCTION}

In drug addicts, exposure to drug-associated cues induces both subjective (ie craving) and behavioral (ie drug seeking) responses, and is thought to play a significant role in the maintenance of the habit, as well as relapse in those attempting to quit (Abrams et al, 1988). Several functional brain-imaging studies have now investigated the neural response to conditioned drug cues in users of various drugs of abuse, including cocaine (Grant et al, 1996; Garavan et al, 2000), alcohol (Tapert et al, 2003), opiates (Sell et al, 2000), and nicotine (Brody et al, 2002; Due et al, 2002; McClernon et al, 2005). In these studies, drug cues elicit activation of neural circuitry thought to encode the motivational and emotional value of the drug, and to play a role in the planning and control of behavior. The brain regions that have been most commonly implicated in cue reactivity include the anterior cingulate cortex (ACC), orbitofrontal

*Correspondence: Dr A Dagher, McConnell Brain Imaging Center, Montreal Neurological Institute, McGill University, 380I University St., Montreal, QC, Canada H3A 2B4, Tel: + I 514398 1726, Fax: + I 514 398 8948, E-mail: alain@bic.mni.mcgill.ca

Received 26 September 2005; revised I February 2006; accepted 27 February 2006

Online publication: 7 March 2006 at http://www.acnp.org/citations/ Npp030706050594/default.pdf cortex (OFC), dorsolateral prefrontal cortex (DLPFC), amygdala, and insula (Wilson et al, 2004). However, two factors that are believed to influence this neural response to drug cues, perceived drug availability and the user's level of abstinence, have seldom been investigated using functional brain imaging. Smokers report a greater urge to smoke when they are anticipating a cigarette (Droungas et al, 1995; Juliano and Brandon, 1998) or during nicotine withdrawal (Shiffman and Jarvik, 1976), and behavioral studies show that the cognitive and autonomic response to smoking cues can be affected by both abstinence (Gross et al, 1993; Johnsen et al, 1997) and expectancy to smoke (Carter and Tiffany, 2001; Wertz and Sayette, 2001).

Based on previous research, we predicted that cueassociated anticipation and planning of imminent drug use would involve the prefrontal cortex (Goldstein and Volkow, 2002; Wilson et al, 2004), in particular the DLPFC, a frontal area important in memory and planning (Petrides, 1994). In a meta-analysis of several cue-induced drug craving studies, Wilson et al (2004) noted that activation of the DLPFC in response to drug cues was observed essentially only in those studies involving subjects not seeking treatment, in which the subjects' expectation to obtain and use the drug after the imaging session could be assumed. Conversely, in all but one of the studies involving individuals undergoing treatment for drug addiction (ie no 
anticipated drug use after the study), there was no DLPFC activation by drug cues. We designed the current study to test the hypothesis that expectation to smoke would modulate the neural response to drug cues, especially in the DLPFC.

Although a recent study failed to find an effect of abstinence on cue-associated functional magnetic resonance imaging (fMRI) activation (McClernon et al, 2005), there is evidence that acute nicotine withdrawal is associated with impairments in brain regions involved in executive function, motivation, and reward (Powell et al, 2002). In particular, smoking withdrawal seems to be associated with a hypo-dopaminergic state in humans (Smolka et al, 2004) and animals (Fung et al, 1996; Epping-Jordan et al, 1998), which could lead to abnormal neural activity in areas implicated in cue-reactivity, in particular the DLPFC, ACC, and striatum.

We therefore used fMRI to investigate brain activity in cigarette smokers exposed to smoking and control videos. The effects of expectancy and abstinence on this neural response were examined by varying the subjects' perceived drug availability and level of withdrawal.

\section{MATERIALS AND METHODS}

\section{Participants}

Twenty healthy regular smokers (10 men and 10 women; mean age $=27$ years, $\mathrm{SD}=8$ ), all smoking at least 15 cigarettes/day $($ mean $=22, \mathrm{SD}=6$ cigarettes/day), were recruited through McGill University classified ads, or by word of mouth. Subjects gave written consent to the study, which was approved by the Montreal Neurological Institute Research Ethics Board, and were monetarily compensated upon completion of the study. The extent of the smoking habit was quantified using the Fagerstrom test for nicotine dependence (Heatherton et al, 1991), in which a minimum score of 5 (out of 10) was necessary for inclusion into the study. Exclusion criteria included any history of psychiatric or neurological illness, previous head trauma, or history of drug abuse (other than nicotine). All subjects but two were right handed.

\section{Study Design}

Each subject was scanned twice, once in an abstinent state (ABS) and once in a non-abstinent state (NON-ABS). The order of scans was randomized and counter-balanced. The $\mathrm{ABS}$ scans required subjects to refrain from smoking for $12 \mathrm{~h}$ prior to the scan whereas for the NON-ABS scans subjects were told to smoke as usual. To minimize any effect of variability in circadian or hormonal cycles on cue reactivity, all scans were performed in early afternoon and each subject's two scans were separated by approximately 4 weeks (mean $=30, S D=5$ days). Compliance with an abstinent state was verified using a carbon monoxide (CO) monitor (Vitalograph, Buckingam, UK), which measures the amount of exhaled CO (in parts per million, p.p.m.) as an indicator of the time since the last cigarette. A CO reading of less than 15 p.p.m. indicated that subjects had been compliant with the abstinence requirement. Subjects were randomly assigned into either the expectant group
(EXP, $n=10$ ) or the nonexpectant group (NON-EXP, $n=10)$. EXP subjects were told that they would be allowed to smoke a cigarette immediately after the scan, whereas NON-EXP subjects were told that they would have to abstain from smoking for $4 \mathrm{~h}$ after the scan (compliance verified by $\mathrm{CO}$ measurement). Subjects in the EXP and NON-EXP groups did not differ significantly in number of years smoking, cigarettes/day, or Fagerstrom test scores. Subjects in the EXP group were on average slightly older than those in the NON-EXP group (EXP: $30, S D=9$; NONEXP: $24, S D=5$ ). Data from one female subject (NON-EXP group) were excluded due to abnormal brain MRI; therefore all results presented are based on data from the remaining 19 subjects.

On the day of the experiment, $30 \mathrm{~min}$ prior to the scan, subjects either smoked a cigarette (NON-ABS scan) or were tested for compliance with a 12-h abstinence (ABS scan). Then, the Profile of Mood States (POMS) (McNair et al, 1992) was administered and subjects were placed on the MRI scanner bed with their head secured using a vacuum bag to minimize movement. Earplugs and headphones were used to lessen scanner noise.

\section{Stimulus Presentation}

Stimulus presentation and response input were coordinated using Media Control Function software (Digivox, Montreal). The behavioral paradigm is represented in Figure 1. Visual stimuli in video format were projected onto a screen at the subjects' feet, and were viewable by means of a mirror mounted on the MRI head coil. Six 2-min videos, alternating between smoking (S) and control (C) content, were presented in the following order: $\mathrm{C}-\mathrm{S}-\mathrm{S}-\mathrm{C}-\mathrm{C}-\mathrm{S}$. Control stimuli consisted of people getting their hair cut, whereas smoking stimuli involved people engaged in smoking behavior (ie lighting cigarettes, smoking while socializing, blowing smoke rings, etc). Young men and women were depicted in each video, and all subjects saw the same video clips. Videos were similar in degree of facial exposure, movement, and physical characteristics of the actors. Different but very similar video clips were shown during the ABS and NON-ABS sessions.

A baseline screen (white cross on a gray background) was displayed for $1 \mathrm{~min}$ prior to the video sequence, and for $20 \mathrm{~s}$ before each video. Subjects responded to a seven item craving questionnaire, taken from a larger battery (Tiffany and Drobes, 1991), using a computer mouse to slide a

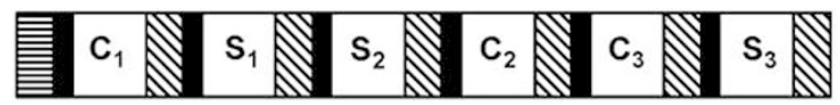

Baseline target screen (1 minute)

Video target screen (20 seconds)

Stimulus video (2 minute)

M Craving questionnaire (1 minute)

Figure I Stimulus presentation scheme. Videos alternated between control (C) and smoking (S) content, with the craving questionnaire following each. 
cursor along a visual analog scale ranging from 0 (not at all) to 10 (extremely). The questions pertained to various aspects of craving, including: general craving (I am craving a cigarette right now), positive aspects of craving (eg 'A cigarette would feel good right now'), negative aspects of craving (eg 'I am feeling irritable right now'), intention to smoke (If it were possible, I would smoke a cigarette right now), and boredom during the previous video (I felt bored during the last video clip). The questionnaire was completed seven times: once before viewing the videos (baseline) and once after each of the six videos.

The stimuli and questionnaire were pilot tested in a separate group of 18 smokers and nine nonsmokers (data not shown). In that study, subjective cigarette craving was increased in smokers after $30 \mathrm{~s}$ and over the entire smoking video, relative to the control video. Positive mood and boredom scores did not differ significantly between smokers and nonsmokers, or between the control and smoking videos. There was an increase in heart rate in nonabstinent smokers $(n=9)$ while watching the smoking video, relative to baseline levels, but not in abstinent smokers $(n=9)$ or nonsmokers.

\section{fMRI Scanning}

All imaging scans were carried out using a 1.5 Tesla Siemens Sonata MRI scanner. Each session began with the acquisition of a series of 160 high-resolution $(1 \mathrm{~mm})$, T1-weighted sagittal images for anatomical localization of the functional data. Blood oxygen level dependent- (BOLD) functional MRI was employed to measure task-dependent neural activity, using a gradient-echo, echo-planar imaging (EPI) sequence (TR, $4000 \mathrm{~ms}$; pause after measurement, $400 \mathrm{~ms}$; TE, $50 \mathrm{~ms}$; flip angle, $90^{\circ}$ ). Each session included one functional scan of approximately $21 \mathrm{~min}$ duration, in which 302 continuous acquisitions were taken. Each acquisition consisted of $36 \mathrm{~T} 2 *$-weighted, contiguous slices (voxel size, $4 \mathrm{~mm} \times 4 \mathrm{~mm} \times 4 \mathrm{~mm}$ ) parallel to the anterior commissureposterior commissure axis and with full brain coverage.

\section{fMRI Data Analysis}

Functional images were blurred with a $6 \mathrm{~mm}$ full-width at half-maximum Gaussian filter and corrected for motion and head drift using the fourth frame as reference (the first three were discarded as the BOLD signal had yet to reach steady state). Frames showing excessive movement were identified visually and excluded from the data set before statistical analysis. Functional BOLD data were analyzed using fmristat software (for full description of statistical method, see Worsley et al (2002); available at http://www.bic.mni.mcgill.ca/users/keith). Signal drift of first-order linear extent was removed from the functional data. In order to combine multiple data sets, both functional EPI images and anatomical images were mapped into standard stereotaxic space using a crosscorrelation algorithm and the Montreal Neurological Institute template (Collins et al, 1994). Subject's individual sessions were first analyzed separately using a fixed effects analysis of the data. All 30 time frames of BOLD data associated with each separate 2-minute control and smoking video segment were averaged, then voxel-wise comparisons between adjacent control and smoking videos (runs) were performed (ie $\mathrm{S}_{1}-\mathrm{C}_{1}, \mathrm{~S}_{2}-\mathrm{C}_{2}$, $\mathrm{S}_{3}-\mathrm{C}_{3}$ ). For each of these pairs of runs and for each subject, an effect magnitude equal to the difference in BOLD between control and smoking video runs, and its SD, were calculated at every voxel by fitting a linear model to the temporal data. This yielded three effect and three SD images per subject per scanning session. These were then combined in two steps, first for each subject, and then across subjects, using a mixed effects linear model to generate t-maps. To determine the overall effect of smoking stimuli on neural activity in cigarette smokers, three t-maps were generated: data were combined for all sessions and subjects (effect of cue for all smokers regardless of condition), across group (effect of cue for EXP and NON-EXP smokers), and across session (effect of cue for ABS and NON-ABS smokers).

T-maps were thresholded for statistical significance using random field theory to correct for multiple comparisons (Worsley et al, 1996; Worsley et al, 2002). Statistical significance was determined based on the peak and extent of each cluster (Poline et al, 1997). A specific hypothesis was made for the involvement of the DLPFC in the neural response to smoking cues during a state of expectancy (Wilson et al, 2004). A DLPFC search region was determined according to the anatomical criteria proposed by Petrides and Pandya (1999) and was composed of the middle frontal gyrus from $y=20$ to $60 \mathrm{~mm}$ (MNI coordinates), including Brodmann's areas (BA) 46 and 9/46 (Petrides and Pandya, 1999), for a total volume of $38000 \mathrm{~mm}^{3}$. Activation within this specified region was deemed significant if the peak voxel exceeded $t=3.88$, which corresponds to $p<0.05$ corrected for multiple comparisons within the search volume. Several brain imaging studies of drug cue-reactivity have shown activations falling within this search volume (Grant et al, 1996; Garavan et al, 2000; Bonson et al, 2002; Brody et al, 2002; Myrick et al, 2004).

Peaks meeting the criterion of statistical significance ( $p<0.05$ corrected) for a whole gray matter search volume of $600 \mathrm{~cm}^{3}$ are also reported. In addition, peaks falling within a priori predicted regions are also listed when they reach significance according to the method of Friston (1997). These regions, which were chosen based on previous cue-induced craving literature, are the ACC, OFC, insula, and limbic system (amygdala, ventral striatum, ventral tegmental area, dorsomedial thalamus). OFC regions are reported but should be interpreted with caution, as there was a significant BOLD signal dropout in this area due to susceptibility artifact.

Regions of interest were drawn for each of the peaks in the smoking - control contrasts. These regions consisted of all contiguous voxels with $t>3.5$ at the peak location. A $2 \times 2$ repeated measures ANOVA was performed on BOLD data extracted from regions of interest, with expectancy as the between-subject factor and abstinence as the withinsubject factor.

For every region of interest, correlations between relative change in BOLD signal and craving scores were performed. All six differences in BOLD and craving scores (smoking - control video) were averaged for each subject (three measures per session, two sessions for each subject) and a stepwise regression between the two measures was performed. To determine if this correlation was modulated 
by expectancy the slopes of the correlation of each group (EXP and NON-EXP) were compared by testing for significance of the interaction product between change in BOLD (dependent variable) and craving $\times$ group (dummy variable). For these correlations we used the score (1-10) to the question: 'I am craving a cigarette right now.'

A voxel-based correlation analysis was performed to determine cue-specific functional connectivity for the ACC because previous work has shown that ACC connectivity is modulated by arousal level (Paus, 2000). The highest peak voxel in the ACC from the subtraction analyses (coordinates: $-4,30,16$, Table 2) was chosen as a seed for the extraction of the BOLD signal time course of each scan. An interaction product was created between this time course and the stimulus contrast. In order to determine functional connectivity modulated by smoking cues, a brain-wide search for voxels where BOLD signal covaried significantly with this interaction was performed, using the stimulus contrasts as confounds. The general linear model equation we used was:

$$
x_{i}=x_{\mathrm{A}} \times S \cdot \beta_{1}+x_{\mathrm{A}} \times C \cdot \beta_{2}+\left[x_{\mathrm{A}} \mathrm{SCD}\right] \cdot \beta_{3}+\varepsilon_{i}
$$

where $x_{i}$ is the BOLD time course at voxel $i, x_{\mathrm{A}}$ the time course at the ACC peak, $S$ and $C$ the smoking and control video designs (convolved with the hemodynamic response function), $D$ is a general confound term which includes signal drift, the $\beta$ are the parameter estimates and the $\varepsilon$ the error. By using the contrast $[1-10]$ we tested for functional connectivity with the seed voxel that was greater during the smoking than the control video. The third term of the equation removes the effects of the task itself and the signal drift. By including the seed voxel activity in this third term we prevent the occurrence of very large correlations at the location of the seed voxel. Effect and SD maps were created for each subject, and then combined across group and session to assess the effect of expectancy and abstinence on functional connectivity. Only regions identified as taking part in the task in the previous smoking minus control analyses were examined.

\section{RESULTS}

\section{Subjective Self-Reports}

No subject had an expired CO level $>15$ p.p.m. prior to the abstinent session (mean: 6.33, SD: 3.16). The CO levels on arrival to the lab for the nonabstinent session were significantly higher (mean: 10.39, SD: 5.69, $p=0.002$ ). Abstinence from smoking significantly decreased selfreported baseline measures of mood and alertness on the POMS. Relative to their nonabstinent session, abstinent subjects reported increased anxiety, depression, fatigue, hostility, insecurity, and confusion (all $p<0.01$ ). Before exposure to the videos, subjects craved cigarettes more when abstinent than nonabstinent (ABS: mean $=5.1$, $\mathrm{SE}=0.3$; NON-ABS: mean $=4.0, \mathrm{SE}=0.3 ; p=0.02$ ); however, there was no significant effect of expectancy on prevideo craving (EXP: mean $=4.3, \mathrm{SE}=0.3$; NON-EXP: mean $=4.8, \quad \mathrm{SE}=0.4 ; \quad p=0.36) . \quad \mathrm{A}$ mixed ANOVA (cue $\times$ abstinence $\times$ expectancy) was performed on the craving self-reports following each video clip for each questionnaire item separately. When compared to the
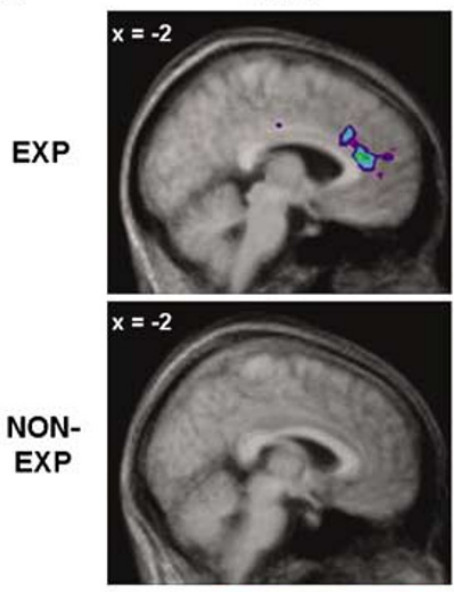

3

b

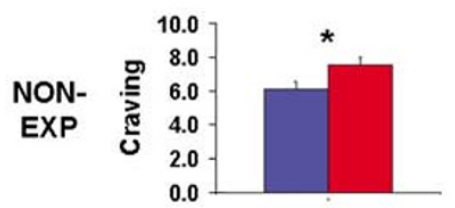

NON-ABS

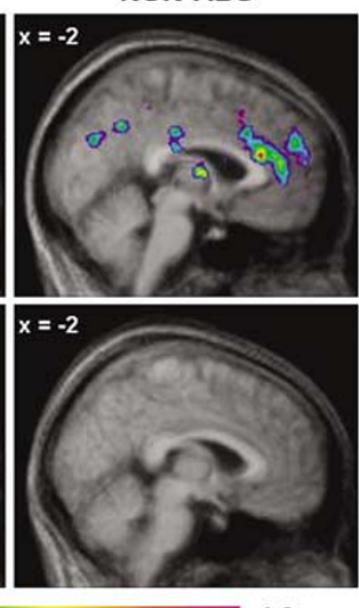

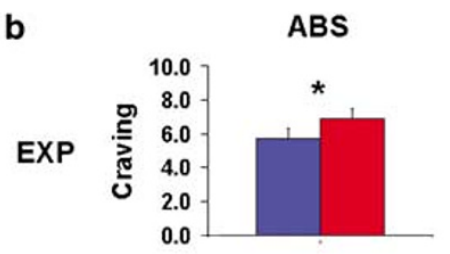
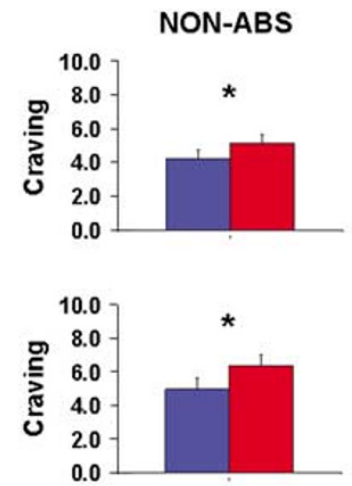

Control
Figure 2 Cue-induced responses in each of the four conditions. (a) Midsagittal t-maps illustrating neural response to smoking cues (relative to control cues) in each condition. Activation to smoking cues was most evident during EXP and NON-ABS. (b) Self-reports to the question: 'I am craving a cigarette right now' after exposure to control and smoking stimuli, in each condition. The craving response to smoking cues (relative to control cues) was statistically similar in all four conditions. ABS, abstinent; NON$\mathrm{ABS}$, nonabstinent; EXP, expectant; NON-EXP, nonexpectant; $* 0<0.01$. Error bars represent SE of the mean.

control videos, smoking videos were successful in significantly increasing subjective reports of craving (main effect of cue; control: mean $=5.2, \mathrm{SE}=0.3$; smoking: mean $=6.5$, $\mathrm{SE}=0.3 ; \mathrm{F}(1,58)=94.5, \quad p<0.001)$, a finding that was evident for each expectancy group and abstinence session (Figure 2). Similar results were seen for all indices of craving, including those addressing positive and negative features, and intention to smoke. Boredom was higher during the control videos than during the smoking videos (control: mean $=5.3, \mathrm{SE}=0.4$; smoking: mean $=3.7$, $\mathrm{SE}=0.3 ; \mathrm{F}(1,58)=35.5, p<0.001)$. While there was an equal increase in craving when comparing smoking to control videos during all sessions, subjects gave overall significantly higher reports of craving when abstinent from cigarettes (main effect of abstinence: $\mathrm{ABS}$ : mean $=6.6$, $\mathrm{SE}=0.2 ; \mathrm{NON}-\mathrm{ABS}:$ mean $=5.2, \mathrm{SE}=0.2 ; \mathrm{F}(1,58)=34.0$, $p<0.001)$. A similar trend existed for other indices, with 
the exception of boredom, which did not differ between abstinence states. There was also a significant main effect of expectancy on reports of craving and boredom during the scan, with nonexpectant subjects reporting higher levels of these two indices than those subjects expecting to smoke after their scan (craving: EXP: mean $=5.5, \quad \mathrm{SE}=0.3$; NON-EXP: mean $=6.3, \quad \mathrm{SE}=0.3 ; \mathrm{F}(1,58)=3.8, \quad p=0.05$; boredom: EXP: mean $=4.1, \mathrm{SE}=0.3$; NON-EXP: mean $=4.9$, $\mathrm{SE}=0.3 ; \mathrm{F}(1,58)=3.9, p=0.05)$. However, there was no significant interaction between cue and either expectancy or abstinence such that the increase in craving in response to smoking cues did not differ between groups or conditions (Figure 2).

\section{BOLD Response to Smoking Stimuli}

All subjects. Table 1 lists brain regions showing BOLD signal change between control and smoking videos for all subjects and conditions combined. Relative to control videos, smoking videos were associated with greater BOLD in left ACC and DMPFC, left medial OFC, left posterior cingulate cortex (PCC), left anterior insula, right superior temporal gyrus, bilateral precuneus, and left dorsomedial thalamus.

The reverse subtraction disclosed greater BOLD during the control videos than the smoking videos in bilateral premotor cortex, supplementary motor area, posterior

Table I Brain Regions of Significant BOLD Signal Contrasts between Neutral and Control Videos for all Subjects and Conditions Combined

\begin{tabular}{|c|c|c|c|c|c|c|c|}
\hline \multirow[b]{2}{*}{ Region } & & \multirow[b]{2}{*}{ Cluster size $\left(\mathrm{mm}^{3}\right)$} & \multicolumn{4}{|c|}{ Peak voxel } & \multirow[b]{2}{*}{ Cluster $p$-value } \\
\hline & & & $x$ & $y$ & $\mathbf{z}$ & t-Value & \\
\hline \multicolumn{8}{|l|}{ Smoking > control } \\
\hline \multirow[t]{2}{*}{ Anterior cingulate cortex (24/32) } & $L$ & 9184 & -4 & 30 & 24 & 5.35 & $<0.001$ \\
\hline & $L$ & & -4 & 40 & 8 & 5.21 & \\
\hline Dorsomedial prefrontal cortex $(9 / 10)$ & $L / R$ & & 0 & 56 & 22 & 4.52 & \\
\hline Medial orbitofrontal cortex $(\mathrm{I} \mid)$ & $L$ & 288 & -8 & 60 & -14 & 4.07 & $0.032^{\mathrm{a}}$ \\
\hline Anterior insula (|3) & $L$ & 1000 & -36 & 12 & 2 & 4.30 & 0.011 \\
\hline Posterior cingulate cortex (23) & $L$ & 1712 & -2 & -16 & 36 & 4.27 & $<0.001$ \\
\hline Superior temporal gyrus $(21 / 22)$ & $\mathrm{R}$ & 2448 & 50 & -34 & 2 & 4.93 & $<0.001$ \\
\hline \multirow[t]{2}{*}{ Precuneus (7) } & $L$ & 968 & -8 & -58 & 36 & 4.60 & 0.013 \\
\hline & $\mathrm{R}$ & & 4 & -58 & 36 & 3.64 & \\
\hline Dorsomedial thalamus & $L$ & 336 & -2 & -6 & 6 & 4.56 & $0.022^{\mathrm{a}}$ \\
\hline \multicolumn{8}{|l|}{ Control > smoking } \\
\hline \multirow{4}{*}{ Precentral gyrus (6) } & $L$ & & -56 & 2 & 34 & 6.65 & $<0.001$ \\
\hline & $\mathrm{R}$ & & 56 & 6 & 35 & 5.60 & \\
\hline & $\mathrm{L}$ & & -26 & -14 & 54 & 7.76 & $<0.001$ \\
\hline & $\mathrm{R}$ & & 26 & -14 & 56 & 8.93 & \\
\hline \multirow[t]{2}{*}{ Posterior insula (I 3) } & $L$ & & -38 & -6 & 16 & 5.92 & 0.002 \\
\hline & $\mathrm{R}$ & & 38 & -2 & 15 & 6.18 & \\
\hline \multirow[t]{2}{*}{ Intraparietal sulcus (40/7) } & $L$ & & -40 & -45 & 58 & 11.08 & $<0.001$ \\
\hline & $\mathrm{R}$ & & 36 & -44 & 52 & 9.12 & \\
\hline \multirow[t]{2}{*}{ Postcentral gyrus (2) } & $\mathrm{L}$ & & -57 & -26 & 38 & 9.71 & \\
\hline & $\mathrm{R}$ & & 54 & -27 & 42 & 8.54 & \\
\hline \multirow[t]{2}{*}{ Superior parietal lobule (7) } & $L$ & & -26 & -58 & 55 & 9.51 & \\
\hline & $\mathrm{R}$ & & 27 & -58 & 58 & 8.08 & \\
\hline \multirow[t]{2}{*}{ Superior occipital gyrus (19) } & $L$ & & -22 & -81 & 38 & 6.97 & \\
\hline & $\mathrm{R}$ & & 25 & -80 & 41 & 7.82 & \\
\hline \multirow[t]{2}{*}{ Middle occipital gyrus (19) } & $L$ & & -33 & -85 & 10 & 6.71 & \\
\hline & $\mathrm{R}$ & & 35 & -82 & 10 & 7.16 & \\
\hline \multirow[t]{2}{*}{ Fusiform gyrus (37) } & $\mathrm{L}$ & & -26 & -52 & -13 & 6.11 & \\
\hline & $\mathrm{R}$ & & 30 & -56 & -13 & 5.53 & \\
\hline \multirow[t]{2}{*}{ Inferior temporal gyrus (19/37) } & $\mathrm{L}$ & & -48 & -68 & -2 & 8.65 & \\
\hline & $\mathrm{R}$ & & 53 & -61 & -6 & 7.95 & \\
\hline
\end{tabular}

L: left; R: right; Numbers in brackets refer to Brodmann's areas; Note: some clusters included more than one anatomical region.

${ }^{a}$ A priori region of interest (Friston, 1997). 
Table 2 Brain Regions of Significant Activation in Response to Smoking Stimuli ( $>$ Control Stimuli) for Expectant $(n=10)$ and NonExpectant $(n=9)$ Groups

\begin{tabular}{|c|c|c|c|c|c|c|c|}
\hline \multirow[b]{2}{*}{ Region } & & \multirow[b]{2}{*}{ Cluster size $\left(\mathrm{mm}^{3}\right)$} & \multicolumn{4}{|c|}{ Peak voxel } & \multirow[b]{2}{*}{ Cluster $p$-value } \\
\hline & & & $x$ & $y$ & $\mathbf{z}$ & t-Value & \\
\hline \multicolumn{8}{|l|}{$\operatorname{EXP}(S>C)$} \\
\hline \multirow[t]{2}{*}{ Anterior cingulate cortex (24/32) } & $L$ & 2688 & -16 & -8 & 26 & 4.82 & $<0.001$ \\
\hline & L & 15976 & -4 & 30 & 16 & 5.82 & $<0.001^{\mathrm{a}}$ \\
\hline \multirow[t]{2}{*}{ Dorsomedial prefrontal cortex $(9 / 10)$} & $L / R$ & & 0 & 58 & 20 & 4.88 & a \\
\hline & $\mathrm{R}$ & & 2 & 32 & 32 & 4.19 & \\
\hline Medial orbitofrontal cortex (I I) & $L$ & 464 & -8 & 60 & -14 & 4.11 & $0.05^{a, b}$ \\
\hline Dorsolateral prefrontal cortex $(9 / 46)$ & $L$ & 248 & -30 & 36 & 42 & 4.19 & $0.015^{\mathrm{c}, \mathrm{d}, \mathrm{e}}$ \\
\hline Dorsolateral prefrontal cortex (9) & $\mathrm{R}$ & 288 & 20 & 58 & 34 & 4.12 & $0.020^{\mathrm{a}, \mathrm{c}}$ \\
\hline Extrastriate visual areas $(18 / 19)$ & $L$ & & -4 & -74 & 26 & 3.65 & \\
\hline Precuneus (7) & L & & -8 & -58 & 36 & 4.52 & $\mathrm{a}, \mathrm{f}$ \\
\hline \multicolumn{8}{|l|}{$N O N-E X P(S>C)$} \\
\hline Superior temporal gyrus (21/22) & $\mathrm{R}$ & 1888 & 54 & -36 & 4 & 4.57 & $<0.001^{g}$ \\
\hline
\end{tabular}

S: BOLD signal associated with smoking cues; C: BOLD signal associated with control cues; L: left; R: right; Numbers in brackets refer to Brodmann's areas; Note: some clusters included more than one anatomical region.

aMain effect of group (EXP $(S>C)>$ NON-EXP $(S>C)) ; p<0.05$.

${ }^{\mathrm{b}} \mathrm{A}$ priori region of interest (Friston, 1997).

'DLPFC a priori region of interest.

'Main effect of group (EXP $(S>C)>$ NON-EXP $(S>C)$ ); $p<0.001$.

eMain effect of group for correlation between self-reports of general craving and BOLD response (EXP $(S>C)>N O N-E X P(S>C)$; $p<0.00 I)$.

'Group-session interaction (EXP/NON-ABS $>$ other conditions; $p=0.03$ ).

gMain effect of group (NON-EXP $(S>C)>\operatorname{EXP}(S>C) ; p=0.03)$.

insula, and a large cluster composed of areas of the postcentral sulcus, intraparietal sulcus, superior parietal lobule, superior and middle occipital gyri, fusiform gyrus, and inferior temporal gyrus. These activations were statistically significant for both expectancy groups and both abstinence sessions.

Expectant vs nonexpectant. Regions activated by smoking stimuli differed between expectancy groups, as demonstrated in Table 2 and Figure 2a. In response to smoking videos (compared to control videos), subjects in the expectant state (abstinent and nonabstinent sessions combined) showed activation of bilateral ACC, DMPFC, and DLPFC (Figure 3a), left medial OFC, left PCC, right posterior parietal cortex, bilateral cuneus, and left precuneus. Subjects in the NON-EXP showed activation only in the right superior temporal gyrus in response to smoking stimuli. When smoking cue-associated activation from regions of interest was contrasted using a $2 \times 2$ ANOVA, the EXP group exhibited significantly higher activation of the $\operatorname{ACC}(\mathrm{F}(1,107)=5.8, p=0.02)$, left DLPFC $(\mathrm{F}=16.7$, $p<0.001)$, right DLPFC $(\mathrm{F}=5.0, p=0.03), \operatorname{DMPFC}(\mathrm{F}=6.3$, $p=0.01)$, left OFC $(\mathrm{F}=4.3, p=0.04)$, left PCC $(\mathrm{F}=4.9$, $p=0.03)$, right posterior parietal cortex $(\mathrm{F}=10.3$, $p=0.002)$, and left precuneus $(F=6.5, p=0.01)$. Nonexpectant smokers showed significantly greater smoking cue-induced activation than expectant smokers in the right superior temporal gyrus $(\mathrm{F}=4.6, p=0.03)$. Figure 4 shows the change in BOLD signal in the ACC and left DLPFC in response to smoking and control videos for each group. There was a significant group by session interaction only in the left precuneus $(\mathrm{F}=4.8, p=0.03)$, with smoking cue-induced activation in this region significantly greater during EXP and NON-ABS than other conditions.

A stepwise linear regression analysis revealed a correlation between smoking cue-induced craving scores and BOLD response in the left DLPFC that was differentially modulated by state of expectancy $(\mathrm{F}(3,17)=20.0, p<0.001$; Figure $3 \mathrm{~b})$. No other significant correlations were seen in any of the areas listed in Tables 1-3.

During the smoking compared to control videos, there was an increase in the correlation between BOLD signal in the left ACC (peak voxel: $x=-4, y=30, z=16$ ) and BOLD signal in the right dorsomedial thalamus $(x=8, y=-12$, $z=4)$ for the EXP group $(t=4.31, p<0.05)$, but not for the NON-EXP group $(t=2.18, \mathrm{NS})$. This effect was statistically different between groups $(t=2.98, p=0.001)$, demonstrat- 

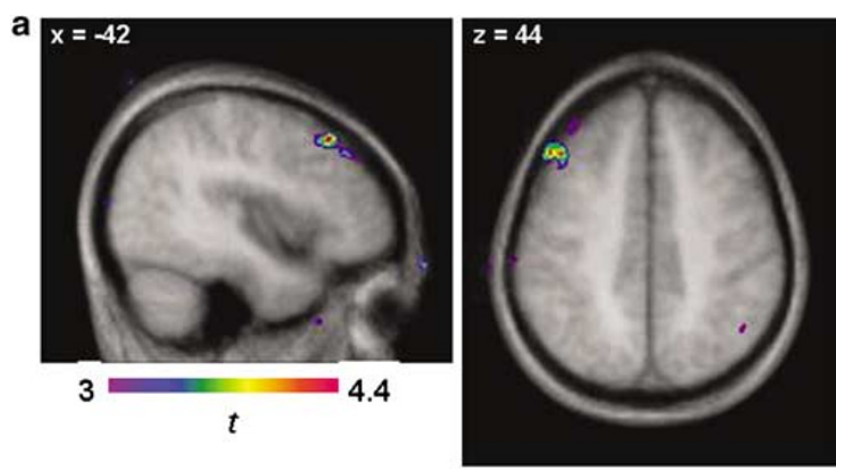

b

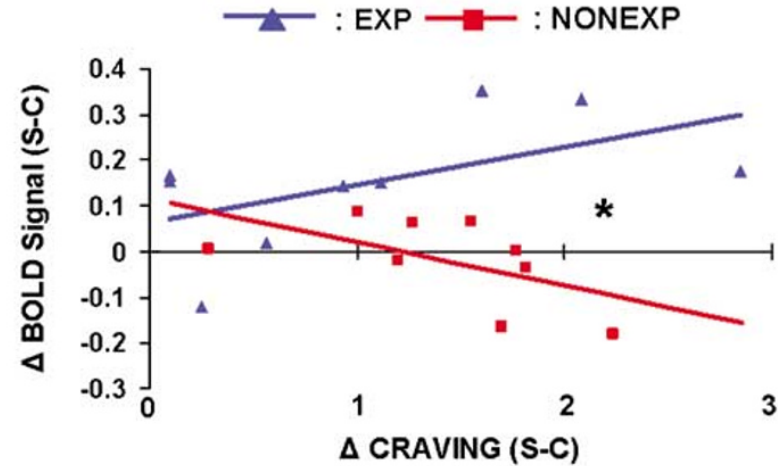

Figure 3 Expectancy and smoking cue-induced response in the dorsolateral prefrontal cortex (DLPFC). (a) T-maps showing activation of the left DLPFC in the expectant group in response to smoking cues (relative to control cues). (b) Correlations between BOLD signal change in the left DLPFC (smoking-control; arbitrary units) and self-reports of general craving (smoking - control) during the expectant and nonexpectant states. The degree of correlation between $\Delta$ BOLD and $\Delta$ craving was statistically different between the EXP and NON-EXP groups, as denoted by the asterisk $(p=0.00 \mathrm{I})$. S, smoking videos; $C$, control videos.

ing that smoking cue-dependent functional connectivity between these two regions during the viewing of the smoking videos was greater for the EXP.

Abstinent vs nonabstinent. The brain response to smoking stimuli (relative to control stimuli) was greater during a non-abstinent state relative to an abstinent state (Table 3 and Figure 2a). Whereas, abstinent smokers (both groups combined) displayed no significant brain response when exposed to smoking cues, smokers in a non-abstinent state showed activation of the left ACC, right PCC, left precuneus, right middle temporal gyrus, and right ventral pallidum extending caudally into the mid-brain tegmentum. However, no brain region showed a significantly different cueinduced BOLD signal change when abstinence states were contrasted.

\section{DISCUSSION}

The main finding in this experiment is that the brain response to drug cues is modulated by expectancy to smoke and, to a lesser extent, level of abstinence. Cigarette smokers responded to smoking stimuli with reports of increased craving, as well as activation of the ACC, PCC, DMPFC, DLPFC, medial OFC, anterior insula, superior temporal gyrus, visuospatial areas, ventral pallidum, and dorsomedial thalamus. While most of these structures have been implicated previously in nicotine cue-reactivity studies (Brody et al, 2002; Due et al, 2002; McClernon et al, 2005), we report here that the neural response to conditioned cues is strongly dependent on perceived drug availability. Indeed, cue-induced activation of the prefrontal, associative and paralimbic regions was observed only in subjects expecting to smoke after the scan.

Consistent with previous imaging studies implicating the PFC in addiction (Goldstein and Volkow, 2002) is the discovery here of an expectancy-dependent $\mathrm{PFC}$ response to drug cues, notably in the ACC, DLPFC, and DMPFC. The DLPFC was activated by smoking cues only in the EXP group, and there was a statistically significant difference in activation between the expectant and nonexpectant subjects. This result confirms a prediction (Wilson et al, 2004) based on the fact that activation of the DLPFC in response to drug cues has been reported essentially only in studies involving subjects not seeking treatment, and who likely expected to use the drug soon after the scanning session.

Because of its involvement in memory (Petrides, 1994), DLPFC activation by drug cues has been proposed to reflect a triggering of memories of drug taking, which could underpin the craving response (Grant et al, 1996). Our results lead to a different interpretation, since we found that equal changes in craving occurred in the presence and absence of DLPFC activation, and that cue-induced activity in the DLPFC correlated positively with craving only in EXP subjects (Figure 3b). Based on our findings, a more likely interpretation of DLPFC involvement in cue reactivity is that it integrates information about internal state (craving, withdrawal), motivation, expectancy, and cues, and uses this information in the regulation and planning of drugseeking or drug-avoiding behavior. DLPFC neuronal activity is known to be modulated by sensory information, motivational state and task contingencies (Miller, 2000), and the DLPFC plays a crucial role in high-level planning of behavior (Passingham, 1993). It is possible that the DLPFC mediates top-down processing of external stimuli (ie drug cues) depending on whether the reward they are associated with (ie the drug) is seen as obtainable. However, one must bear in mind that our methodology is only sensitive to relatively acute changes in signal. Sustained DLPFC activity that might accompany more enduring craving responses, due for example to the abstinent state, or to drug-taking ruminations or memories induced by the video clips, would not be detectable by our block design fMRI paradigm.

The medial frontal lobe (ACC and DMPFC) showed the most widespread response to smoking stimuli, but once again, activation was dependent on the expectancy state. Moreover, the ACC or DMPFC signal change did not correlate with self-reports of craving, consistent with previous experiments involving smokers (Brody et al, 2002) as well as cocaine (Wexler et al, 2001) and opiate addicts (Daglish et al, 2001). Furthermore, the NON-EXP group had the same cue-related increases in craving as the EXP group and higher average craving reports during the scanning session despite failing to demonstrate any frontal activation to the cues (Table 2). We note, however, that other cue-reactivity imaging studies have uncovered a correlation between craving and ACC activation (Maas 

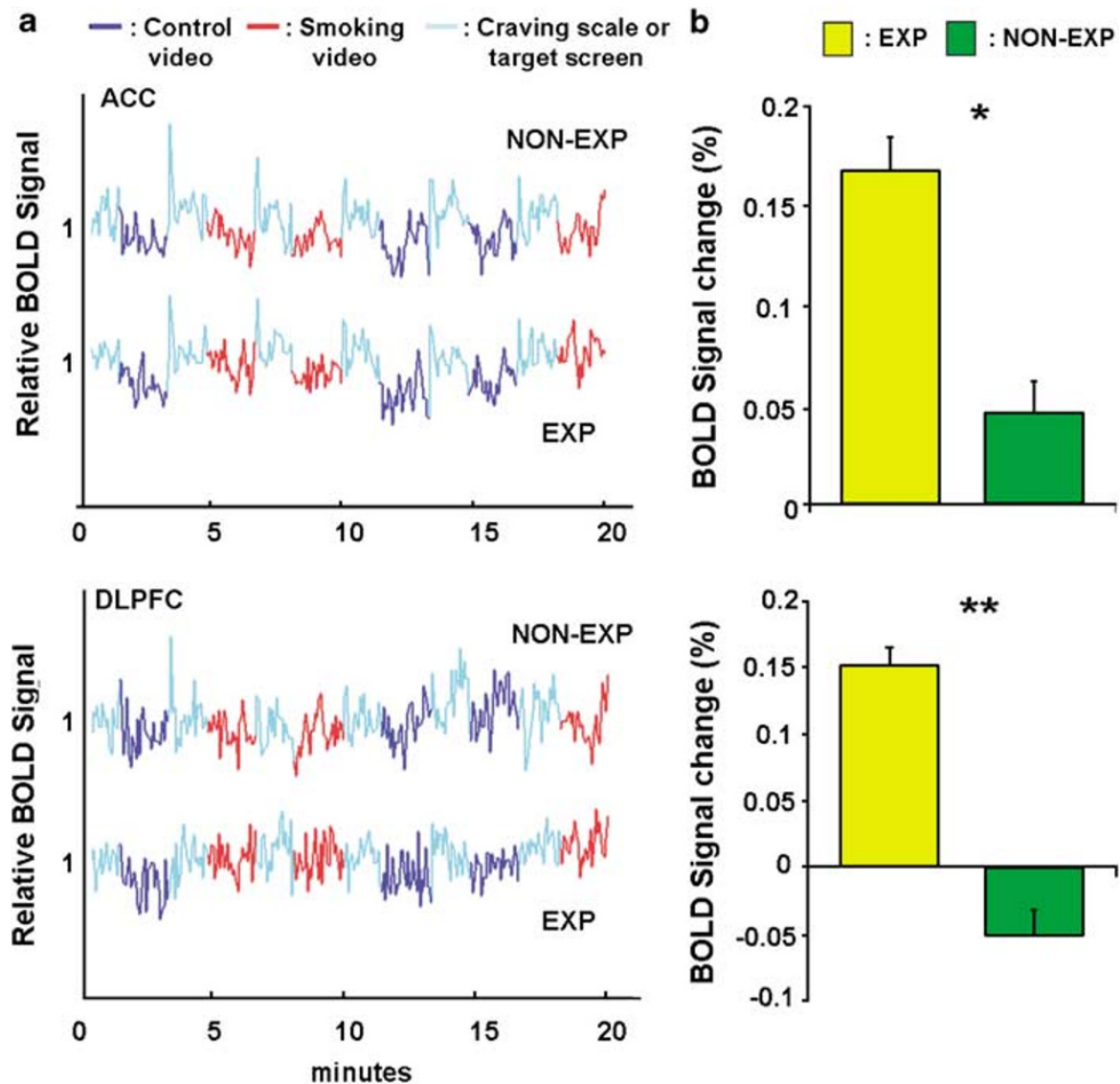

Figure 4 BOLD signal response to control and smoking stimuli for left anterior cingulate cortex (ACC, top) and left dorsolateral prefrontal cortex (DLPFC, bottom). (a) Timecourses of relative BOLD signal throughout entire stimulus presentation. For each expectancy group, ACC and DLPFC time courses represent BOLD data from all voxels in the cluster exceeding a $t$-value of 3.5, for all subjects and sessions combined. (b) Corresponding graphs of percent BOLD signal change from control to smoking stimuli in each condition for the ACC (top) and DLPFC (bottom). Note the greater smoking cueassociated signal for the EXP group (relative to NON-EXP) in these regions. EXP, expectant; NON-EXP, nonexpectant. Error bars represent SEM. * $p<0.05 ; * * * 0.001$

Table 3 Brain Regions of Significant Activation in Response to Smoking Stimuli (>Control Stimuli) for Abstinent $(n=19)$ and NonAbstinent $(n=19)$ Sessions

Peak voxel

\begin{tabular}{|c|c|c|c|c|c|c|c|}
\hline \multirow[b]{2}{*}{ Region } & & \multirow[b]{2}{*}{ Cluster size $\left(\mathrm{mm}^{3}\right)$} & & \multirow[b]{2}{*}{ Cluster $p$-value } \\
\hline & & & $x$ & $y$ & $\mathbf{z}$ & t-Value & \\
\hline \multicolumn{8}{|l|}{$A B S(S>C)$} \\
\hline No significant peaks & - & - & - & - & - & - & - \\
\hline \multicolumn{8}{|l|}{ NON-ABS $(S>C)$} \\
\hline Anterior cingulate cortex (24/32) & $\mathrm{L}$ & 4696 & -2 & 28 & 18 & 4.57 & $<0.001$ \\
\hline Precuneus (3l/7) & $\mathrm{L}$ & 864 & -8 & -58 & 36 & 3.94 & 0.022 \\
\hline Middle temporal gyrus $(2 \mathrm{I})$ & $\mathrm{R}$ & 728 & 50 & -32 & 2 & 4.14 & 0.046 \\
\hline
\end{tabular}

S: BOLD signal associated with smoking cues; C: BOLD signal associated with control cues; L: left; R: right; Numbers in brackets refer to Brodmann's areas.

et al, 1998; Myrick et al, 2004; Brody et al, 2004; McClernon et al, 2005).

What could account for the effect of expectancy on ACC activation? Several theories of ACC function have been proposed, most commonly invoking a role in attention and arousal. Paus et al (1998) argue that supracallosal ACC activation across a wide range of cognitive and emotional tasks is due to engagement of nonspecific arousal systems 
that project from the brainstem via the thalamus to the ACC. Our finding of an increase in functional connectivity between the ACC and dorsomedial thalamus when comparing the smoking to the control videos, at coordinates identical to those identified in previous studies of arousal (Hofle et al, 1997; Paus, 2000), suggests that smoking cues act on thalamo-cortical arousal systems. Moreover, this cuerelated increase in thalamo-cingulate connectivity was only present in the EXP, suggesting that the effect of cues on arousal was greater in the expectant state, a finding consistent with behavioral studies (Carter and Tiffany, 2001). Indeed, our subjects reported less boredom during exposure to smoking cues (relative to control cues), and during the expectant state. We therefore suggest that perceived availability increases the arousing effects of drug cues, a process likely involving the ACC.

We also observed cue-related activation, greater in the EXP than the NON-EXP, in posterior visual areas including precuneus (BA 7) and posterior parietal areas (BA 7, 40), which likely indicates top-down modulation of visual areas by frontal attentional systems (Desimone and Duncan, 1995). Indeed, the ACC region activated in our study is also implicated in shifting visual attention (Gitelman et al, 1999). Thus, the ACC and DLPFC may act to make smoking cues more salient, an important factor in promoting drug use (Robinson and Berridge, 1993). Our study suggests that this attentional bias toward smoking cues is greater in the expectant state, which is consistent with previous behavioral studies (Wertz and Sayette, 2001).

The ACC activation extended anterior into the DMPFC (BA 9, 10), but once again only in the EXP group. The DMPFC has been associated with states when 'attention is directed specifically toward self-referential or introspectively oriented mental activity' (Gusnard et al, 2001). While focused attention usually leads to reductions in activity in DMPFC, instructions designed to direct the subject's attention toward their current emotional state have the opposite effect. Gusnard et al (2001) asked subjects to view a set of pictures and to pay attention either to how the pictures made them feel or to whether they were taken indoors. They found greater BOLD signal during the monitoring of emotions in the same DMPFC area identified in our study. In both their experiment and ours, subjects viewed the same stimuli while in two mental states that differed only due to the instructions given to the subjects (expectation to smoke in our case), and in both cases this contingency was associated with differential response in DMPFC.

Abstinence from smoking was associated with a relatively blunted neural response to smoking cues (Table 3). Nicotine withdrawal is known to result in a hypoactive dopamine system (Fung et al, 1996; Epping-Jordan et al, 1998), which could explain why we found the ventral pallidum, an important meso-limbic dopamine projection site (Klitenick et al, 1992), to be activated by smoking stimuli only in the NON-ABS session. Higher plasma levels of nicotine during the nonabstinent scans could also explain the greater neural response to smoking cues in this state, since nicotine has a facilitatory effect on the dopaminergic response to salient stimuli (Rice and Cragg, 2004; Zhang and Sulzer, 2004). However, as no brain region showed a statistically significant difference in activity in response to drug cues when abstinence states were contrasted, further investigation will be necessary to determine how withdrawal interacts with conditioned cues. A recent fMRI study failed to detect a statistically significant effect of abstinence level on cue-induced fMRI activation (McClernon et al, 2005).

We also observed consistent bilateral increases in signal in a set of brain regions in response to the control videos (compared to smoking) throughout all sessions, regardless of expectancy or abstinence state, consisting of extrastriate visual, posterior parietal, and lateral premotor areas (Table 1). This may be a consequence of observing tool manipulations during the neutral (haircut) videos (Buccino et al, 2001), although it could also represent suppression of neural activity during the smoking videos, as previously demonstrated for posterior parietal areas in subjects viewing emotional material (Lane et al, 1997; Gusnard et al, 2001).

Our results can be contrasted with two recent nicotine cue-reactivity imaging studies. In one, smokers undergoing smoking cessation treatment with the drug bupropion were compared to regular smokers not attempting to quit in a positron emission tomography study employing both visual cues and actual cigarettes (Brody et al, 2004). The untreated smokers had a significantly greater increase in glucose metabolism in the ACC than the bupropion group, when comparing smoking to neutral cues. Since the bupropion subjects were attempting to quit (and had either managed to quit or significantly reduced their intake since starting the medication), they may be compared to our NON-EXP group, while the untreated smokers were identical to our EXP group. If one accepts this, the findings of this study are identical to ours, and the reduction in ACC activation (at coordinates very close to ours) in the treated group can be attributed to a lack of expectancy (and possibly desire) to smoke.

In another study, a procedure resembling ours was used. Two groups underwent fMRI scanning while being exposed to cigarette and neutral cues (Wilson et al, 2005). The groups differed only in expectation to smoke after the scan, and subjects were randomly assigned to each group. Only one prefrontal area showed activation solely in the EXP group: the medial OFC (coordinates very close to ours). Surprisingly, the DLPFC failed to be activated in either group, while the ACC was equally activated in both groups. There were significant differences in study design however: the cue was an actual cigarette, the expectant subjects were expecting to smoke immediately $(40 \mathrm{~s})$ after the cigarette cue exposure, and the total duration of exposure to the cues was shorter (74 s compared to $360 \mathrm{~s}$ in our study).

A key finding in our experiment is the context-dependent dissociation between the subjective reaction to smoking stimuli and the concurrent fMRI response (Figure 2). Smokers reported equal increases in craving during exposure to the smoking videos (compared to the control videos) in all four experimental states. However, a cueassociated increase in BOLD signal in areas previously implicated in craving was only seen in the expectant state. This incongruity likely reveals a fundamental difference between the measured neural and self-reported subjective responses to drug cues. According to cognitive theories of cue-reactivity (Tiffany, 1990), subjective craving reports represent active, nonautomatic interpretations of an inter- 
nal state, whereas the autonomic and neural responses to the drug cues are generated by more automatic processing that drives drug seeking. Indeed, our data failed to show a simple relationship between the neural activity that results from exposure to conditioned cues and the craving that they provoke.

These results are subject to caution however. There is no consensus on how to define or measure drug craving (Drummond, 2001). Self-report measures of craving are particularly prone to errors due to influence of the experimental context (ie subjects 'know' that the cues are supposed to make them crave), self-deception, variable interpretation of scales or questions, and differences in response style (Sayette et al, 2000), and may therefore not measure 'true craving'. Moreover, the temporal dynamics of craving are complex. It is likely that in our study, craving lasted longer than the duration of cue exposure, and that it was also affected by factors other than the drug cues, such as nicotine withdrawal, habituation to the cues, and the stress of the procedure.

Nonetheless, the neural response to drug cues was seen in brain areas involved in arousal, attention, motivation, and executive control, which is consistent with the theory that exposure to these cues leads to drug seeking or relapse independently of craving (Tiffany, 1990).

In conclusion, this study demonstrated that the neural response to conditioned drug cues is dependent on the user's level of expectancy. The interaction of cues and anticipation to consume determines neural activity in brain regions that are known to play a role in the seeking and consumption of the drug.

\section{ACKNOWLEDGEMENTS}

We are grateful to Isabelle Boileau, Mike Ferreira, and Bojana Stefanovic for their assistance, to Tomas Paus for helpful discussions, and to the Canadian Institutes for Health Research and the Canadian Tobacco Control Research Initiative.

\section{REFERENCES}

Abrams DB, Monti PM, Carey KB, Pinto RP, Jacobus SI (1988). Reactivity to smoking cues and relapse: two studies of discriminant validity. Behav Res Ther 26: 225-233.

Bonson KR, Grant SJ, Contoreggi CS, Links JM, Metcalfe J, Weyl HL et al (2002). Neural systems and cue-induced cocaine craving. Neuropsychopharmacology 26: 376-386.

Brody AL, Mandelkern MA, Lee G, Smith E, Sadeghi M, Saxena S et al (2004). Attenuation of cue-induced cigarette craving and anterior cingulate cortex activation in bupropion-treated smokers: a preliminary study. Psychiatr Res 130: 269-281.

Brody AL, Mandelkern MA, London ED, Childress AR, Lee GS, Bota RG et al (2002). Brain metabolic changes during cigarette craving. Arch Gen Psychiatr 59: 1162-1172.

Buccino G, Binkofski F, Fink GR, Fadiga L, Fogassi L, Gallese V et al (2001). Action observation activates premotor and parietal areas in a somatotopic manner: an fMRI study. Eur J Neurosci 13: $400-404$.

Carter BL, Tiffany ST (2001). The cue-availability paradigm: the effects of cigarette availability on cue reactivity in smokers. Exp Clin Psychopharmacol 9: 183-190.
Collins DL, Neelin P, Peters TM, Evans AC (1994). Automatic 3D intersubject registration of MR volumetric data in standardized Talairach space. J Comput Assist Tomogr 18: 192-205.

Daglish MR, Weinstein A, Malizia AL, Wilson S, Melichar JK, Britten $S$ et al (2001). Changes in regional cerebral blood flow elicited by craving memories in abstinent opiate-dependent subjects. Am J Psychiatr 158: 1680-1686.

Desimone R, Duncan J (1995). Neural mechanisms of selective visual attention. Annu Rev Neurosci 18: 193-222.

Droungas A, Ehrman RN, Childress AR, O’Brien CP (1995). Effect of smoking cues and cigarette availability on craving and smoking behavior. Addict Behav 20: 657-673.

Drummond DC (2001). Theories of drug craving, ancient and modern. Addiction 96: 33-46.

Due DL, Huettel SA, Hall WG, Rubin DC (2002). Activation in mesolimbic and visuospatial neural circuits elicited by smoking cues: evidence from functional magnetic resonance imaging. $\mathrm{Am}$ J Psychiatr 159: 954-960.

Epping-Jordan MP, Watkins SS, Koob GF, Markou A (1998). Dramatic decreases in brain reward function during nicotine withdrawal. Nature 393: 76-79.

Friston KJ (1997). Testing for anatomically specified regional effects. Hum Brain Mapp 5: 133-136.

Fung YK, Schmid MJ, Anderson TM, Lau YS (1996). Effects of nicotine withdrawal on central dopaminergic systems. Pharmacol Biochem Behav 53: 635-640.

Garavan H, Pankiewicz J, Bloom A, Cho JK, Sperry L, Ross TJ et al (2000). Cue-induced cocaine craving: neuroanatomical specificity for drug users and drug stimuli. Am J Psychiatr 157: 17891798.

Gitelman DR, Nobre AC, Parrish TB, LaBar KS, Kim YH, Meyer JR et al (1999). A large-scale distributed network for covert spatial attention: further anatomical delineation based on stringent behavioural and cognitive controls. Brain 122(Part 6): 1093-1106.

Goldstein RZ, Volkow ND (2002). Drug addiction and its underlying neurobiological basis: neuroimaging evidence for the involvement of the frontal cortex. Am J Psychiatr 159: $1642-1652$.

Grant S, London ED, Newlin DB, Villemagne VL, Liu X, Contoreggi C et al (1996). Activation of memory circuits during cue-elicited cocaine craving. Proc Natl Acad Sci USA 93: 12040-12045.

Gross TM, Jarvik ME, Rosenblatt MR (1993). Nicotine abstinence produces content-specific Stroop interference. Psychopharmacology (Berlin) 110: 333-336.

Gusnard DA, Akbudak E, Shulman GL, Raichle ME (2001). Medial prefrontal cortex and self-referential mental activity: relation to a default mode of brain function. Proc Natl Acad Sci USA 98: $4259-4264$.

Heatherton TF, Kozlowski LT, Frecker RC, Fagerstrom KO (1991). The Fagerstrom Test for Nicotine Dependence: a revision of the Fagerstrom Tolerance Questionnaire. Br J Addict 86: 1119-1127.

Hofle N, Paus T, Reutens D, Fiset P, Gotman J, Evans AC et al (1997). Regional cerebral blood flow changes as a function of delta and spindle activity during slow wave sleep in humans. J Neurosci 17: 4800-4808.

Johnsen BH, Thayer JF, Laberg JC, Asbjornsen AE (1997). Attentional bias in active smokers, abstinent smokers, and nonsmokers. Addict Behav 22: 813-817.

Juliano LM, Brandon TH (1998). Reactivity to instructed smoking availability and environmental cues: evidence with urge and reaction time. Exp Clin Psychopharmacol 6: 45-53.

Klitenick MA, Deutch AY, Churchill L, Kalivas PW (1992). Topography and functional role of dopaminergic projections from the ventral mesencephalic tegmentum to the ventral pallidum. Neuroscience 50: 371-386.

Lane RD, Fink GR, Chau PM, Dolan RJ (1997). Neural activation during selective attention to subjective emotional responses. Neuroreport 8: 3969-3972. 
Maas LC, Lukas SE, Kaufman MJ, Weiss RD, Daniels SL, Rogers VW et al (1998). Functional magnetic resonance imaging of human brain activation during cue-induced cocaine craving. Am J Psychiatr 155: 124-126.

McClernon FJ, Hiott FB, Huettel SA, Rose JE (2005). Abstinenceinduced changes in self-report craving correlate with eventrelated FMRI responses to smoking cues. Neuropsychopharmacology 30: 1940-1947.

McNair D, Lorr M, Droppleman L (1992). EITS Manual for Profile of Mood States. Educational and Industrial Testing Service: San Diego.

Miller EK (2000). The prefrontal cortex and cognitive control. Nat Rev Neurosci 1: 59-65.

Myrick H, Anton RF, Li X, Henderson S, Drobes D, Voronin K et al (2004). Differential brain activity in alcoholics and social drinkers to alcohol cues: relationship to craving. Neuropsychopharmacology 29: 393-402.

Passingham RE (1993). The Frontal Lobes and Voluntary Action. Oxford University Press: Oxford.

Paus T (2000). Functional anatomy of arousal and attention systems in the human brain. Prog Brain Res 126: 65-77.

Paus T, Koski L, Caramanos Z, Westbury C (1998). Regional differences in the effects of task difficulty and motor output on blood flow response in the human anterior cingulate cortex: a review of 107 PET activation studies. Neuroreport 9: R37-R47.

Petrides M (1994). Frontal lobes and working memory: evidence from investigations of the effects of cortical excisions in nonhuman primates. In: Boller F, Grafman J (eds). Handbook of Neuropsychology. Elsevier: Amsterdam. pp 59-82.

Petrides M, Pandya DN (1999). Dorsolateral prefrontal cortex: comparative cytoarchitectonic analysis in the human and the macaque brain and corticocortical connection patterns. Eur $J$ Neurosci 11: 1011-1036.

Poline JB, Worsley KJ, Evans AC, Friston KJ (1997). Combining spatial extent and peak intensity to test for activations in functional imaging. Neuroimage 5: 83-96.

Powell J, Dawkins L, Davis RE (2002). Smoking, reward responsiveness, and response inhibition: tests of an incentive motivational model. Biol Psychiatr 51: 151-163.

Rice ME, Cragg SJ (2004). Nicotine amplifies reward-related dopamine signals in striatum. Nat Neurosci 7: 583-584.

Robinson TE, Berridge KC (1993). The neural basis of drug craving: an incentive-sensitization theory of addiction. Brain Res Brain Res Rev 18: 247-291.
Sayette MA, Shiffman S, Tiffany ST, Niaura RS, Martin CS, Shadel WG (2000). The measurement of drug craving. Addiction 95(Suppl 2): S189-S210.

Sell LA, Morris JS, Bearn J, Frackowiak RS, Friston KJ, Dolan RJ (2000). Neural responses associated with cue evoked emotional states and heroin in opiate addicts. Drug Alcohol Depend 60: 207-216.

Shiffman SM, Jarvik ME (1976). Smoking withdrawal symptoms in two weeks of abstinence. Psychopharmacology (Berlin) 50: 35-39.

Smolka MN, Budde H, Karow AC, Schmidt LG (2004). Neuroendocrinological and neuropsychological correlates of dopaminergic function in nicotine dependence. Psychopharmacology (Berlin) 175: 374-381.

Tapert SF, Cheung EH, Brown GG, Frank LR, Paulus MP, Schweinsburg AD et al (2003). Neural response to alcohol stimuli in adolescents with alcohol use disorder. Arch Gen Psychiatr 60: 727-735.

Tiffany ST (1990). A cognitive model of drug urges and drug-use behavior: role of automatic and nonautomatic processes. Psychol Rev 97: 147-168.

Tiffany ST, Drobes DJ (1991). The development and initial validation of a questionnaire on smoking urges. $\mathrm{Br}$ J Addict 86: $1467-1476$.

Wertz JM, Sayette MA (2001). Effects of smoking opportunity on attentional bias in smokers. Psychol Addict Behav 15: 268-271.

Wexler BE, Gottschalk CH, Fulbright RK, Prohovnik I, Lacadie CM, Rounsaville BJ et al (2001). Functional magnetic resonance imaging of cocaine craving. Am J Psychiatr 158: 86-95.

Wilson SJ, Sayette MA, Delgado MR, Fiez JA (2005). Instructed smoking expectancy modulates cue-elicited neural activity: a preliminary study. Nicotine Tob Res 7: 637-645.

Wilson SJ, Sayette MA, Fiez JA (2004). Prefrontal responses to drug cues: a neurocognitive analysis. Nat Neurosci 7: 211-214.

Worsley K, Marrett S, Neelin P, Vandal A, Friston K, Evans A (1996). A unified statistical approach for determining significant signals in images of cerebral activation. Hum Brain Mapp 4: 58-73.

Worsley KJ, Liao CH, Aston J, Petre V, Duncan GH, Morales F et al (2002). A general statistical analysis for fMRI data. Neuroimage 15: $1-15$.

Zhang H, Sulzer D (2004). Frequency-dependent modulation of dopamine release by nicotine. Nat Neurosci 7: 581-582. 